\title{
Lattice QCD with 8 and 12 degenerate quark flavors
}

\author{
Xiao-Yong Jin* \\ Department of Physics, Columbia University, New York, NY 10027, USA \\ E-mail: xj2106@columbia.edu
}

\section{Robert D. Mawhinney}

Department of Physics, Columbia University, New York, NY 10027, USA

E-mail: rdmephysics.columbia.edu

\begin{abstract}
We compare extensive simulations of QCD with 8 and 12 flavors of degenerate quarks, using the DBW2 gauge action, naive staggered fermions, and the rational hybrid Monte Carlo algorithm. A variety of values of the coupling constant, quark mass, and lattice size have been used. Our data suggests that, as the bare coupling is decreased, a rapid cross-over, which dramatically changes the lattice scale, exists with both 8 and 12 flavors. The scale change across this cross-over is much larger with 12 flavors than it is with 8 flavors. All of the observables we have measured, in both the zero and finite temperature systems, are consistent with a chiral symmetry breaking phase for the zero temperature theory on the weak coupling side of the rapid cross-over.
\end{abstract}

The XXVII International Symposium on Lattice Field Theory

July 26 - 31, 2009

Peking University, Beijing, China

\footnotetext{
* Speaker.
} 

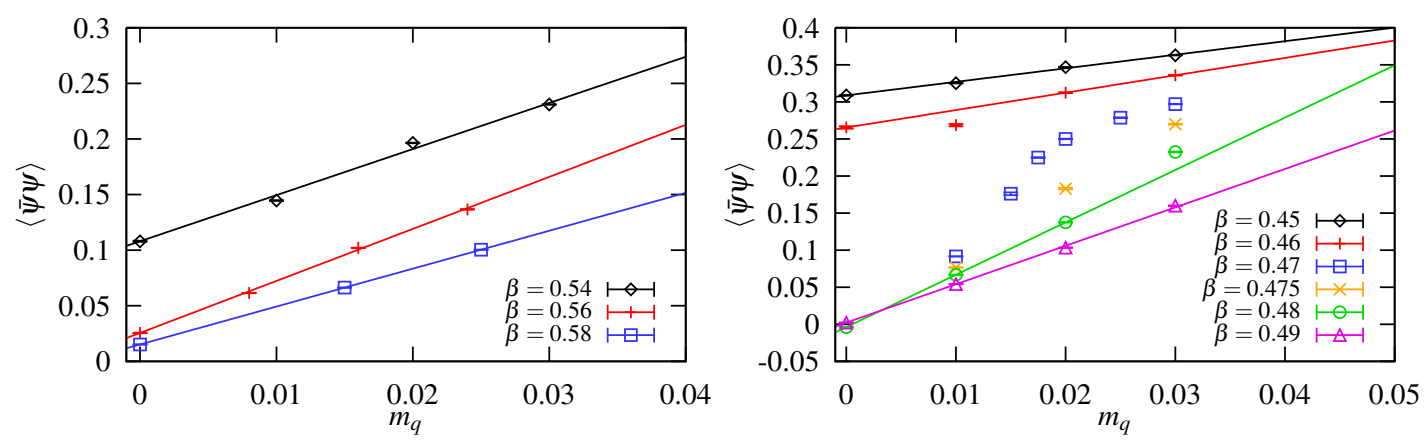

Figure 1: The chiral condensate for 8 flavors (left panel) with $\langle\bar{\psi} \psi\rangle\left(\beta=0.56, m_{q}=0\right)=0.02555(23)$. The right panel is for 12 flavors, where $\langle\bar{\psi} \psi\rangle\left(\beta=0.49, m_{q}=0\right)=0.00220(27)$

\section{Zero temperature simulations}

In this paper, we report results from numerical simulations of QCD with 12 flavors, which extend our previous investigation of 8-flavor QCD [1]. We seek evidence for the phase of zero temperature QCD with these values of $N_{f}$, which is relevant to locating a possible infrared fixed point and/or walking behavior. We approach the problem directly by measuring standard hadronic observables such as meson propagators and masses, the pion decay constant, the static heavy-quark potential, and the string tension in zero temperature lattice simulations.

As in our earlier, 8-flavor study [1], we have used the DBW2 gauge action and naive staggered fermion action for our study of 12-flavor QCD. The DBW2 action reduces flavor symmetry breaking [1] while still being fast in simulations. The RHMC algorithm is used here, even though we do not require fractional powers of the fermion determinant, since this version of our code is the most efficient. Measurements are separated by 10 trajectories, each of which has a length of 1/2 MD time units. Additionally, we have done an 8-flavor simulation with larger volume at our smallest mass and weakest coupling to check finite volume effects and have seen no changes.

Table 1 is a list of our major simulations with 12 flavors at $N_{\tau}=32$. We have done ordered and disordered starts on the smallest lattices for all parameters, to check for a unique phase for each parameter choice, and both starts produce the same thermalized values for the chiral condensate, indicating no metastability. Generally 600-800 time units are used for thermalization and then measurements are made every 10th time unit for a total of $\sim 150$ measurements. By simulating with multiple volumes, we see that finite volume effects in $m_{\pi}$ and $m_{\rho}$ are under control up to $\beta=0.475$, but that larger volumes are needed at $\beta=0.49$. The results from $\beta=0.50$ are not used in the analysis here, because the volumes are too small. The string tension shows larger finite volume effects, which may be due to our measurement method. In this paper, results from the largest volume available are used in the analysis.

Fig. 1 shows plots of the chiral condensate for both 8 and 12 flavors. The 8 -flavor graph on the left, from 3 equally spaced $\beta$ values, shows a large change in $\langle\bar{\psi} \psi\rangle\left(m_{q}=0\right)$ with $\beta$. Linear extrapolations in $m_{q}$ give a non-zero value for $\langle\bar{\psi} \psi\rangle\left(m_{q}=0\right)$, so the theory is in the chirally broken phase. For 12 flavors (right graph), a transition from strong coupling to weak coupling is seen, and in the transition region, non-linear quark mass dependence is visible. We see that for $\beta \lesssim 0.45$ the system is clearly in a chirally broken phase, from the non-zero value of $\langle\bar{\psi} \psi\rangle\left(m_{q}=0\right)$. For 


\begin{tabular}{|c|c|c|c|c|c|c|}
\hline$\beta$ & $m_{f}$ & Size & $\langle\bar{\psi} \psi\rangle$ & $m_{\pi}$ & $m_{\rho}$ & $\sigma$ \\
\hline \multirow{3}{*}{0.45} & "0.01 & $16^{3} \times 32$ & $0.32521(26)$ & $0.25327(51)$ & & \\
\hline & 0.02 & $16^{3} \times 32$ & $0.34687(17)$ & $0.35376(34)$ & & \\
\hline & 0.03 & $16^{3} \times 32$ & $0.36290(16)$ & $0.42867(28)$ & & \\
\hline \multirow{3}{*}{0.46} & 0.01 & $16^{3} \times 32$ & $0.2687(17)$ & $0.26430(47)$ & & \\
\hline & 0.02 & $16^{3} \times 32$ & $0.31240(57)$ & $0.36137(35)$ & & \\
\hline & 0.03 & $16^{3} \times 32$ & $0.33586(12)$ & $0.43617(22)$ & & \\
\hline \multirow{6}{*}{0.47} & \multirow{3}{*}{0.01} & $16^{3} \times 32$ & $0.09047(51)$ & $0.3159(11)$ & $0.662(19)$ & $0.0471(31)$ \\
\hline & & $24^{3} \times 32$ & $0.09108(36)$ & $0.31465(61)$ & $0.671(83)$ & $0.0432(40)$ \\
\hline & & $32^{3} \times 32$ & $0.09157(15)$ & $0.31497(34)$ & $0.678(17)$ & $0.04309(92)$ \\
\hline & \multirow{2}{*}{0.02} & $16^{3} \times 32$ & $0.25025(47)$ & $0.37743(42)$ & $1.192(17)$ & $0.377(39)$ \\
\hline & & $24^{3} \times 32$ & $0.24995(37)$ & $0.37787(35)$ & $1.2289(58)$ & $0.393(26)$ \\
\hline & 0.03 & $16^{3} \times 32$ & $0.29695(25)$ & $0.44691(31)$ & $1.295(46)$ & $0.407(70)$ \\
\hline \multirow{4}{*}{0.475} & \multirow{2}{*}{0.01} & $16^{3} \times 32$ & $0.07819(73)$ & $0.3182(14)$ & $0.625(17)$ & $0.0446(22)$ \\
\hline & & $24^{3} \times 32$ & $0.07651(23)$ & $0.31721(79)$ & $0.5839(46)$ & $0.0315(12)$ \\
\hline & 0.02 & $16^{3} \times 32$ & $0.1830(11)$ & $0.39947(51)$ & $1.064(10)$ & $0.100(21)$ \\
\hline & 0.03 & $16^{3} \times 32$ & $0.26986(50)$ & $0.45500(29)$ & $1.328(15)$ & $0.372(58)$ \\
\hline \multirow{6}{*}{0.48} & \multirow{3}{*}{0.01} & $16^{3} \times 32$ & $0.06560(48)$ & $0.3190(17)$ & $0.5369(87)$ & $0.0279(26)$ \\
\hline & & $24^{3} \times 32$ & $0.06662(12)$ & $0.31253(41)$ & $0.5247(44)$ & $0.0224(14)$ \\
\hline & & $32^{3} \times 32$ & $0.066839(61)$ & $0.31456(53)$ & $0.5308(32)$ & $0.02265(53)$ \\
\hline & \multirow{2}{*}{0.02} & $16^{3} \times 32$ & $0.13642(53)$ & $0.41539(55)$ & $0.894(15)$ & $0.0875(28)$ \\
\hline & & $24^{3} \times 32$ & $0.13750(22)$ & $0.41586(52)$ & $0.8952(94)$ & $0.0776(39)$ \\
\hline & 0.03 & $16^{3} \times 32$ & $0.23246(55)$ & $0.46619(45)$ & $1.1700(51)$ & $0.269(19)$ \\
\hline \multirow{4}{*}{0.49} & \multirow{2}{*}{0.01} & $16^{3} \times 32$ & $0.05326(67)$ & $0.3293(37)$ & $0.5231(75)$ & $0.0255(16)$ \\
\hline & & $32^{3} \times 32$ & $0.05420(12)$ & $0.30573(69)$ & $0.4497(37)$ & $0.0130(14)$ \\
\hline & 0.02 & $16^{3} \times 32$ & $0.10335(34)$ & $0.4230(10)$ & $0.7353(60)$ & $0.0547(24)$ \\
\hline & 0.03 & $16^{3} \times 32$ & $0.15994(45)$ & $0.49419(41)$ & $1.006(13)$ & $0.1056(55)$ \\
\hline \multirow{3}{*}{0.50} & 0.01 & $16^{3} \times 32$ & $0.04420(11)$ & $0.3579(29)$ & $0.5336(95)$ & $0.0189(12)$ \\
\hline & 0.02 & $16^{3} \times 32$ & $0.08816(19)$ & $0.4166(15)$ & $0.6352(97)$ & $0.0399(22)$ \\
\hline & 0.03 & $16^{3} \times 32$ & $0.12876(17)$ & $0.49931(69)$ & $0.836(14)$ & $0.0708(23)$ \\
\hline
\end{tabular}

Table 1: Simulation parameters and some results for 12-flavor QCD.

$\beta \gtrsim 0.49$, we will argue that the system is still in a chirally broken phase, but with a much smaller scale (in lattice units) than the theory with $\beta \lesssim 0.45$.

We will refer to the region between $\beta \simeq 0.54$ and $\simeq 0.58$ (for 8 flavors) as $\Delta \beta_{8}$ and the region from $\beta \simeq 0.46$ and $\simeq 0.49$ (for 12 flavors) as $\Delta \beta_{12}$. In this range of $\beta$ values, both systems are showing rapid changes, and we want to determine the phase of the theory on the weak coupling side of this rapid evolution region.

Throughout this report, we will be using simple analytic extrapolations to $m_{q}=0$. For QCDlike theories in the chirally broken phase, there will be chiral logarithms in such an extrapolation. A priori one does not have any understanding of the quark mass range over which chiral logs might be seen, since this requires knowing the size of the chiral limit decay constant, $f$, and the chiral 

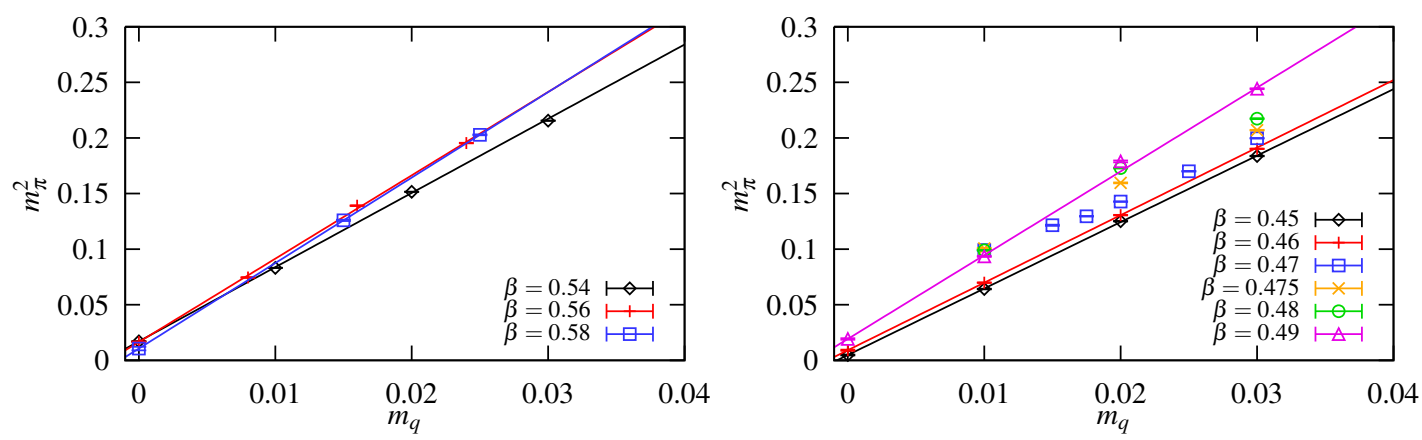

Figure 2: A plot of $m_{\pi}^{2}$ versus $m_{q}$ for 8 flavors (left panel) with $m_{\pi}^{2}\left(\beta=0.56, m_{q}=0\right)=0.01648(51)$. The right panel is for 12 flavors with $m_{\pi}^{2}\left(\beta=0.45, m_{q}=0\right)=0.00482(39)$ and $m_{\pi}^{2}\left(\beta=0.49, m_{q}=0\right)=$ $0.01926(66)$.

condensate. In addition, explicit factors of $N_{f}$ multiply chiral logarithms, further influencing the quark mass range where they are visible. In $2+1$ flavor QCD, where much work has been done on this topic, simple linear extrapolations are rather accurate and we will employ them here, but it is important to remember that we are making this ansatz.

Fig. 2 shows $m_{\pi}^{2}$ versus $m_{q}$. For 8 flavors, we can see clearly that $m_{\pi}^{2}=2 B m_{q}$, the behavior expected for a Goldstone boson. $B$ changes little with $\beta$ and the intercept is small. (It is important to include chiral logarithms in an extrapolation to test that $m_{\pi}=0$ when $m_{q}=0$.) For 12 flavors, at strong coupling, the same Goldstone behavior is seen. In the $\Delta \beta_{12}$ region the situation is not as clear. For weaker coupling, $\beta=0.49$, we see noticeable finite volume effects in $m_{\pi}$ at the smallest quark mass, $m_{q}=0.01$. (Finite volume effects are known to effect the extrapolation of $m_{\pi}^{2}$ to $m_{q}=0$ [2].) Since we have a larger volume for $m_{q}=0.01$, we use this result for $m_{\pi}$ in our fit and this gives a small intercept for $N_{f}=12$ as shown in the right panel of Fig. 2. Thus we have a good evidence for the pion, with 12 flavors, to be a Goldstone boson. This implies a non-zero dimensionful parameter, $B$, which also argues against a conformal phase. We note that for 12 flavors, $B$ also has a mild dependence on $\beta$.

The pion decay constant, $f_{\pi}$, for 8 and 12 flavors is shown in Fig. 3 . We can see that $f_{\pi}$ in the chiral limit changes by about about $2 \times$ for 8 flavors, across the $\Delta \beta_{8}$ region, and by about $10 \times$ for 12 flavors. Again we see evidence for a non-zero dimensionful parameter, $f_{\pi}$, on the weak coupling side of the $\Delta \beta_{12}$ region. We can use the Gell-Mann-Oakes-Renner relation, $\langle\bar{\psi} \psi\rangle \propto m_{\pi}^{2} f_{\pi}^{2} / m_{q}$, to predict $\langle\bar{\psi} \psi\rangle$ for 12 flavors. We have seen that $m_{\pi}^{2} / m_{q}$ is almost independent of $\beta$, which means that $\langle\bar{\psi} \psi\rangle \propto f_{\pi}^{2}$ on both sides of the $\Delta \beta_{12}$ region. This gives a $100 \times$ change in $\langle\bar{\psi} \psi\rangle$ across the $\Delta \beta_{12}$ region. This is consistent with our direct measurements of $\langle\bar{\psi} \psi\rangle$ from Fig 1, since the $100 \times$ change in $\langle\bar{\psi} \psi\rangle$ makes it likely to be too small to determine well in our current simulations.

We can also investigate the scale change in the string tension, $\sigma$, as shown in Fig. 4. Being a dimension two quantity, $\sigma$ should scale as $f_{\pi}^{2}$. It is easily seen for 8 flavors to change by roughly $4 \times$. For 12 flavors, Fig. 4 shows $\sigma \approx 0.35$ in the chiral limit for $\beta=0.47$, found by linearly fitting the heavier quarks with $m_{q}=0.02,0.025,0.03$. (Lighter quarks are in the rapid transition region and measuring $\sigma$ at smaller $\beta$ values is difficult because it is large.) A $10 \times$ change in scale would give $\sigma \approx 0.0035$ on the weak coupling side of the $\Delta \beta_{12}$ region, which is consistent, within errors, with what we can measure. 

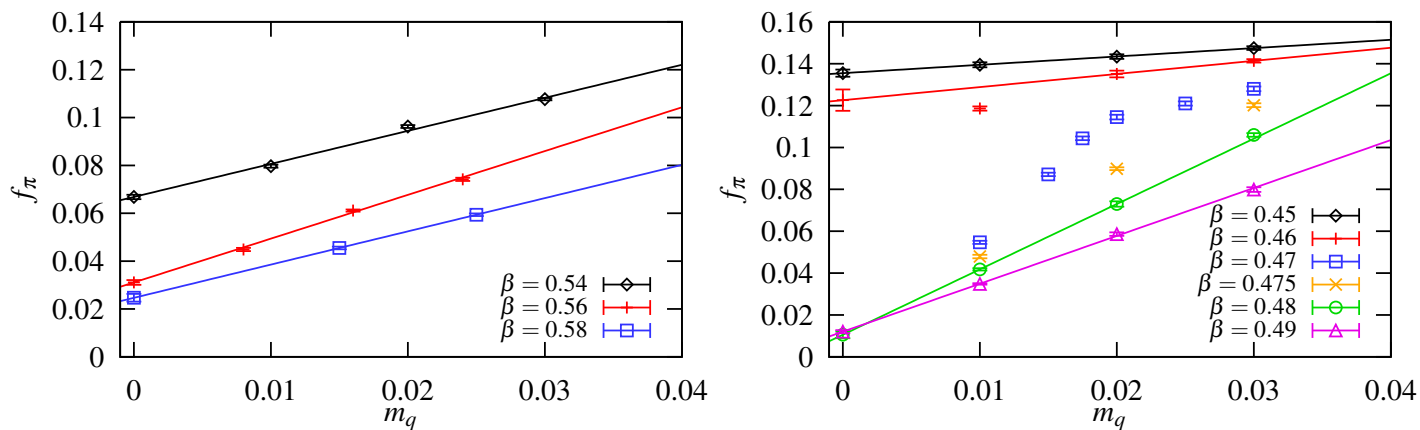

Figure 3: The pion decay constant for 8 flavors (left panel) and for 12 flavors (right panel).
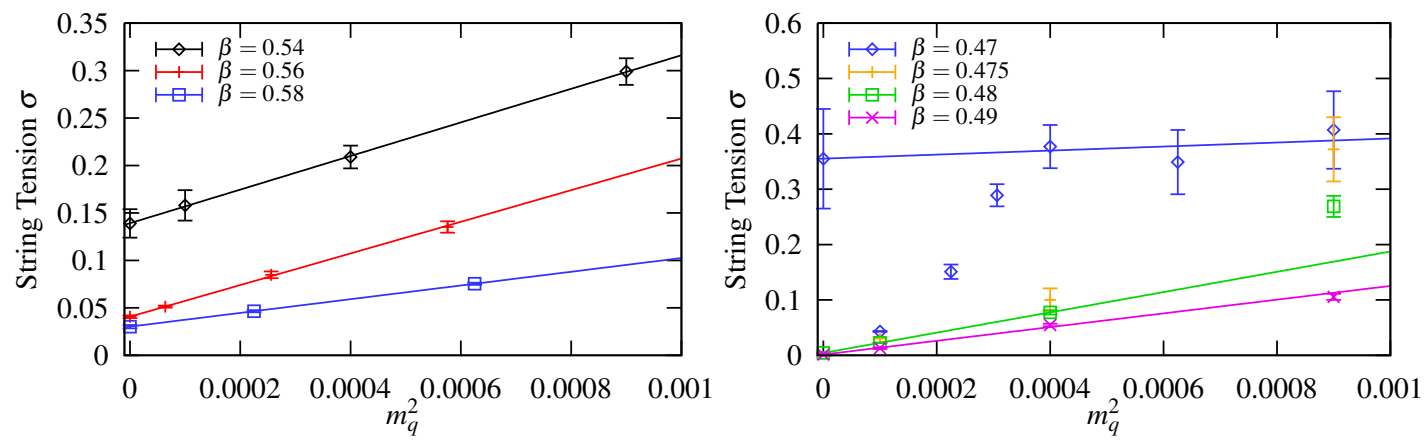

Figure 4: The string tension versus $m_{q}^{2}$ for 8 flavors (left panel) and 12 flavors (right panel), with $\sigma(\beta=$ $\left.0.48, m_{q}=0\right)=0.0043(15)$, and $\sigma\left(\beta=0.49, m_{q}=0\right)=0.0014(17)$.
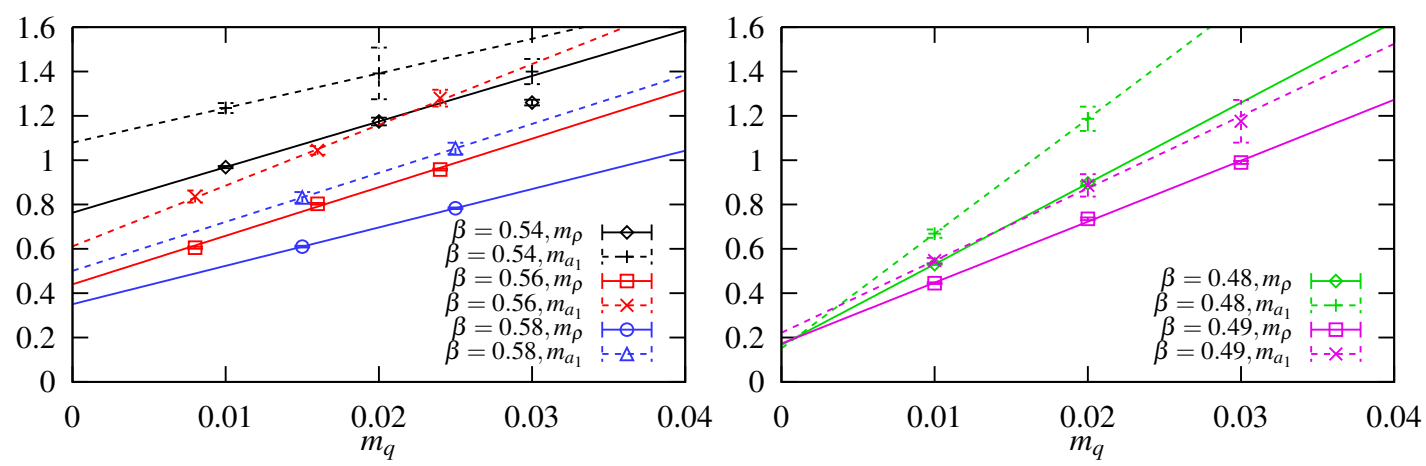

Figure 5: The spectrum of the parity partners, $\rho$ and $a_{1}$. The left panel is for 8 flavors and the right for 12 flavors. Dashed lines are linear extrapolation of $m_{a_{1}}$. Solid lines are linear extrapolation of $m_{\rho}$.

It was observed [3] that, for 4 flavors, the spectrum of parity partners, like the $\rho$ and $a_{1}$, becomes degenerate if the volume is too small, even if the theory is still in the broken chiral symmetry phase. The masses of the $\rho$ and $a_{1}$ are shown in Fig. 5, where, for 12 flavors, only values at the two weakest coupling are shown, because the larger masses that result from stronger coupling are hard to extract. 8-flavor QCD shows no visible parity doubling, while for 12 flavors parity doubling is clearly visible, suggesting finite volume effects are present in our simulations of the chirally broken 12 flavor theory. 

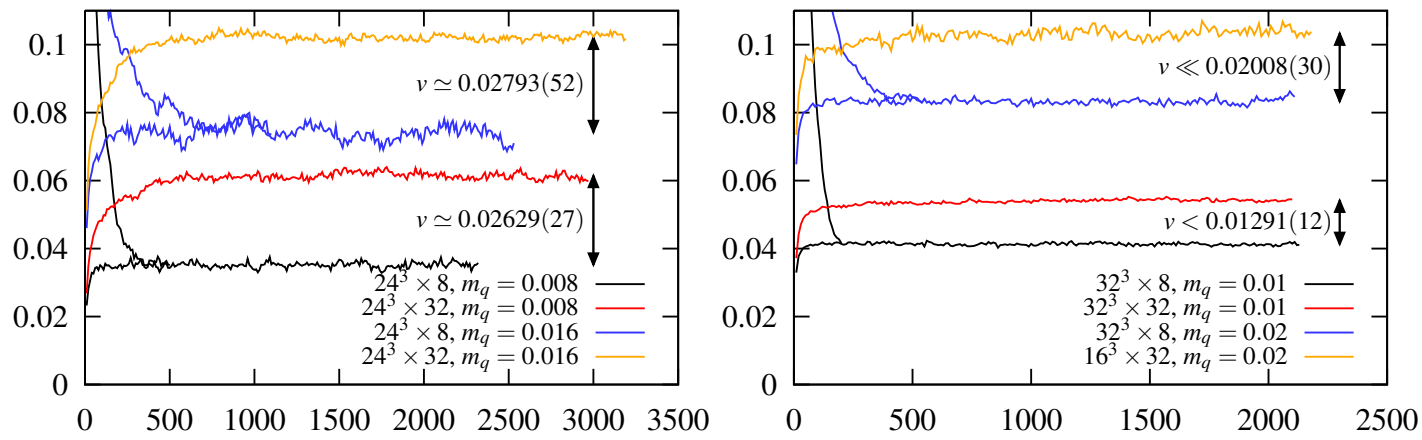

Figure 6: A comparison of the evolutions of $\langle\bar{\psi} \psi\rangle$ between $N_{\tau}=8$ and $N_{\tau}=32$. The left panel is for 8 flavors and the right panel is for 12 . Both ordered and disordered starts are shown for $N_{\tau}=8$, but only an ordered start is shown for $N_{\tau}=32$.
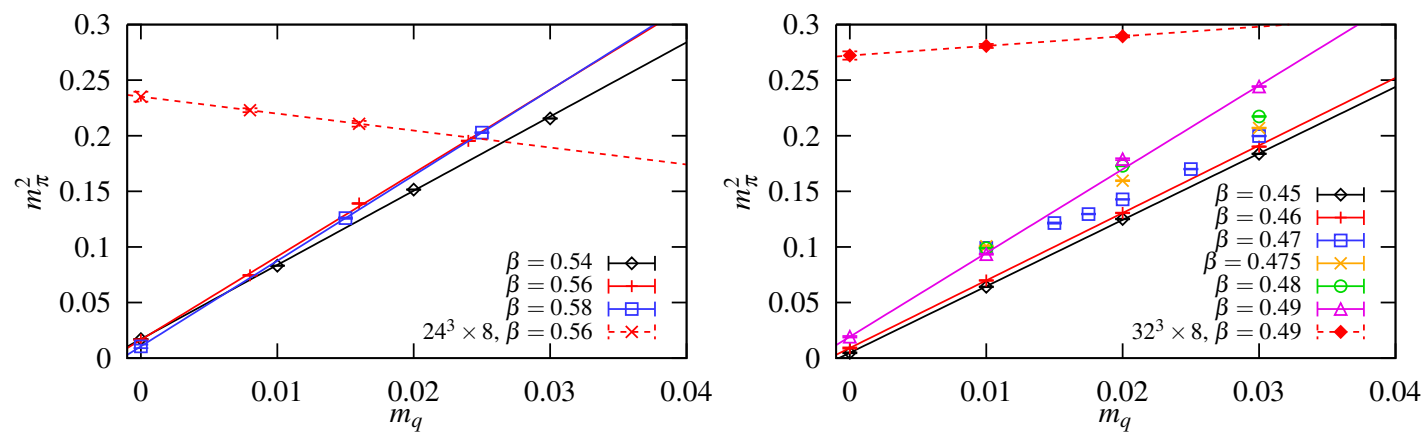

Figure 7: $m_{\pi}^{2}$ versus $m_{q}^{2}$. Dashed lines are screening mass at $N_{\tau}=8$. Left panel is 8 flavors. Right panel is 12 flavors.

\section{Finite temperature simulations}

We have presented considerable evidence that 12 flavors is in a chirally broken phase. A check of these arguments is to run at higher temperature and look for evidence of chiral symmetry restoration. After the conference, we did finite temperature simulations at $\beta=0.56$ with a lattice size of $24^{3} \times 8$ for 8 flavors, and at $\beta=0.49$ with a lattice size of $32^{3} \times 8$ for 12 flavors. The evolutions of $\langle\bar{\psi} \psi\rangle$ are shown in Fig. 6. The amount the chiral condensate drops at finite $T$ should be equal to the $T=0$ vacuum expectation value which we have determined. This is easily seen to be the case for 8 flavors, but for 12 flavors, we do not have an accurate measurement of this $T=0$ quantity. Also, with 12 flavors, the finite temperature change in $\langle\bar{\psi} \psi\rangle$ varies with quark mass, consistent with the $T=0$ simulations not being entirely on the weak coupling side of the $\Delta \beta_{12}$ region.

A clearer signal comes from the screening mass of the pion, measured along a spatial direction with $N_{\tau}=8$. This is shown in Fig. 7. At fixed $\beta$, we have changed $N_{\tau}$ from 32 to 8 , and seen a clear, dramatic change in the behvior of $m_{\pi}$ with $m_{q}$. Obviously, it is no longer a Goldstone boson. We have also measured the Polyakov loop along the temporal direction for both $N_{\tau}=8$ and 32. It is clearly non zero at $N_{\tau}=8$, and vanishes within errors for $N_{\tau}=32$. We conclude that there is a thermal transition taking place between $N_{\tau}=8$ and $N_{\tau}=32$ for both 8 flavors and 12 flavors. 


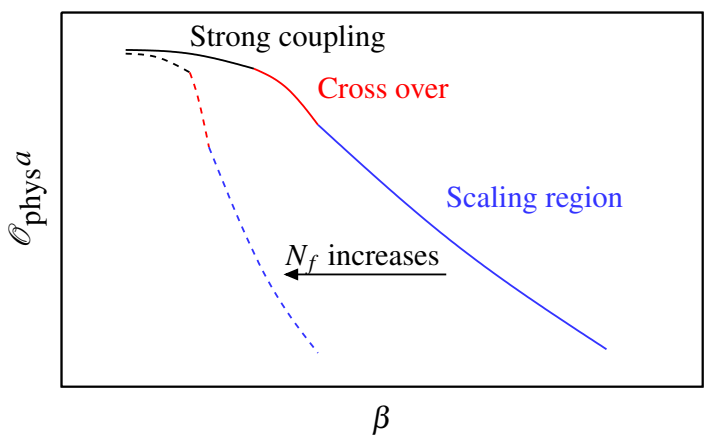

Figure 8: Generic dependence of an observable with $\beta$.

\section{Conclusion}

We have presented considerable evidence that QCD with 8 and 12 flavors is in a chiral symmetry breaking phase. We see a pion which behaves as a Goldstone boson for $T=0$ and which does not for finite $T$. Multiple dimensionful quantities are found, with $m_{q}=0$, when $T=0$, inconsistent with a conformal phase.

There is clearly a marked change in the system with 12 flavors, which we discuss in the context of Fig. 8, which shows how an observable in lattice units changes with $\beta$. Previous $N_{f}=8$ studies [4] argued that the cross-over could become a phase transition, due to lattice artifacts. With our DBW2 action, this is no longer seen, rather we have a smooth, somewhat rapid, change away from strong coupling. This change becomes considerably more rapid for $N_{f}=12$, but the system still breaks chiral symmetry. Currently, we do not know if our simulations at weaker coupling are in the cross-over region, or at weak enough coupling to begin to see the continuum, scaling region of the system. Our rapid change for $N_{f}=12$ could be an indicator of walking behavior (as expected for this large number of flavors), or just a steepened cross-over region still influenced by lattice artifacts.

We are thankful to all members of the RBC collaboration and especially to Norman Christ for insightful discussions. Our calculations were done on the QCDOC at Columbia University and NY Blue at BNL. This research utilized resources at the New York Center for Computational Sciences at Stony Brook University/Brookhaven National Laboratory which is supported by the U.S. Department, of Energy under Contract No. DE-FG02-92ER40699 and by the, State of New York.

\section{References}

[1] X.-Y. Jin and R. D. Mawhinney, Lattice QCD with Eight Degenerate Quark Flavors, PoS LATTICE2008 (2008) 059 [arXiv:0812.0413 [hep-lat]].

[2] C.-Z. Sui, Flavor dependence of quantum chromodynamics, . UMI-99-98219.

[3] R. D. Mawhinney, Review of unquenched results, Nucl. Phys. Proc. Suppl. 83 (2000) 57-66 [arXiv:hep-lat/0001032].

[4] F. R. Brown et al., Lattice QCD with eight light quark flavors, Phys. Rev. D46 (1992) 5655-5670 [arXiv:hep-lat/9206001]. 